\title{
ESTUDO EXPLORATÓRIO SOBRE REALIDADE AUMENTADA E LABORATÓRIO REMOTO NO ENSINO DE FÍSICA
}

\author{
Priscila Cadorin Nicolete, PPGIE, UFRGS, priscilanicolete@hotmail.com \\ Eduardo Tocchetto de Oliveira Júnior, IFSC, du.tocchetto@gmail.com \\ Marta Adriana Cristiano, UFSC, marta.php@gmail.com \\ Liane Margarida Rockenbach Tarouco,PPGIE, UFRGS, liane@penta.ufrgs.br \\ Eduardo de Vila, UFSC, eduardodevila1@hotmail.com \\ Juarez Bento da Silva, PPGTIC, UFSC, juarezbs.silva@gmail.com
}

\begin{abstract}
Resumo. Este estudo busca investigar se o uso de Laboratórios Remotos Aumentados e Laboratórios Virtuais de Realidade Aumentada contribui para o ensino de circuitos elétricos aos alunos de ensino médio técnico em Eletromecânica. Por meio de uma pesquisa exploratória, de caráter explicativo, abordagem mista e utilizando um design quase-experimental, foi verificado a percepção dos estudantes em relação a motivação, engajamento, usabilidade e aprendizagem percebida. Além de verificar a progressão de saberes dos estudantes, por meio de uma avaliação diagnóstica e um avaliação conceitual. Os resultados apresentaram significativo progresso na compreensão do conteúdo explorado.
\end{abstract}

Palavras-chave: Laboratório Remoto, Realidade Aumentada, Tecnologia educacional

\section{EXPLORATORY STUDY ON AUGMENTED REALITY AND REMOTE LAB IN PHYSICS TEACHING}

\begin{abstract}
This paper aims to investigate the use or use of Augmented Remote Laboratories and Augmented Virtual Reality Lab contributed to some of the electrical circuits to some of the technical medium in Electromechanical. Through an exploratory research and mixed approach and using a quasi-experimental design, it was verified the students' perception regarding motivation, engagement, usability and perceived learning. In addition to verifying the progress of knowledge of two students, by means of a diagnostic assessment and a joint assessment. The results show significant progress in the understanding of the scanned content.
\end{abstract}

Keywords: Remote Lab, Augmented Reality, Educational Technology.

\section{Introdução}

A ausência de práticas no ensino de disciplinas STEM (Ciência, Tecnologia, Engenharia e Matemática) pode dificultar a assimilação da teoria com a realidade vivida pelo estudante. Em consequência, isso pode levar a dificuldades para compreender uma série de analogias e inferências necessárias à abstração das leis científicas. Entretanto, apesar da sua importância, a experimentação nem sempre é uma realidade presente nas salas de aula. O que é visto é, usualmente, um ensino pautado em aulas excessivamente expositivas, nas quais os alunos assumem um papel passivo em sua aprendizagem.

A falta de experimentação, muitas vezes, está relacionada às dificuldades que os professores enfrentam diante da infraestrutura das instituições de ensino para esse V. $17 \mathrm{~N}^{\mathrm{o}}$ 3, dezembro, 2019 DOI:

RENOTE 
tipo de atividade. Segundo dados do Censo Escolar 2018, apenas 11\% das escolas de ensino básico possuem laboratórios de ciências. Em relação às escolas públicas, apenas $8 \%$ possuem esse tipo de laboratório, e mesmo quando existentes são por vezes precários (Inep/Mec, 2018).

Do outro lado, a complexidade e diversidade que caracterizam a sociedade contemporânea traduz as relevantes mudanças no âmbito tecnológico no cotidiano das pessoas. Indubitavelmente o elevado grau de complexidade, dinamismo e integração dos mais diversos tipos de conhecimentos influenciam a educação em todos os seus níveis. Nota-se que a educação precisa caminhar para a informatização, se aproximando de seus alunos - nativos digitais - inserindo-os em um ambiente tecnológico que ofereça acesso a recursos como Laboratórios Virtuais (LV) e Laboratórios Remotos (LR), tecnologia de Realidade Aumentada (RA), entre outros (Rodrigues et al., 2017).

A RA, participante ativa deste estudo, é um sistema que complementa o mundo real com objetos gerados por computador alinhando-os de forma interativa e em tempo real (Azuma et al., 2001). Estudada desde meados dos anos 90, a RA tem ganhado destaque nos últimos anos, principalmente pela popularização de dispositivos portáteis que permitem o acesso simplificado a esse tipo de recurso. E é neste contexto, que se potencializa como ferramenta de apoio nos processos de ensino e aprendizagem, fornecendo ferramentas que permitem a validação do aprendizado teórico (Frank; Kapila, 2017).

Com o intuito de explorar todo o potencial que as tecnologias podem oferecer para educação, os Laboratórios Remotos incorporam a RA ampliando os processos de ensino e aprendizagem apoiados em laboratório online, uma vez que essas tecnologias juntas são capazes de conectar o mundo real do laboratório remoto e o mundo virtual dos laboratórios virtuais, moldando novos ambientes educacionais (Zandavi; Chung, 2018). O Laboratório Remoto Aumentado ${ }^{1}$, produz um valor agregado para a experimentação remota, aumentando a sensação de presença e realidade.

Diante deste paradigma, emerge a questão sobre a percepção dos estudantes em termos de experiência de uso e de aprendizagem após o uso de um Laboratório Remoto Aumentado (LRA) e um Laboratório Virtual de RA (LVRA). Para responder a isto, desenvolveu-se um Laboratório Remoto Aumentado e um Laboratório Virtual de RA e elaborou-se uma pesquisa exploratória, cujo principal objetivo consiste em investigar a percepção dos estudantes após o uso dos laboratórios para o ensino de circuitos elétricos em uma turma do ensino médio técnico em Eletromecânica.

\section{O uso de Realidade Aumentada em combinação com laboratórios remotos}

Atualmente, a linha que diferencia laboratórios remotos e virtuais está ficando cada vez mais tênue devido ao surgimento de laboratórios que possuem características de ambas tecnologias. Assim, surgem os laboratórios híbridos que são formas avançadas de laboratórios que, não só imitam experiências práticas, mas também fornecem novos recursos, criando possibilidades diferentes das encontradas nos laboratórios tradicionais. Os laboratórios híbridos, basicamente, misturam elementos virtuais e remotos na tentativa de aproveitar as vantagens proporcionadas por cada um desses, a fim de oferecer realismo, custo-benefício e recursos adicionais.

Nessa perspectiva, Mejías, Andújar e Sánchez (2014) propõem o conceito de Laboratório Remoto Aumentado (LRA), que utiliza RA para criação de laboratórios

\footnotetext{
1 Termo cunhado por Andujar, Mejias e Marquez (2011) para denominar laboratórios remotos que utilizam técnicas de realidade aumentada. 
híbridos. Conforme os autores, a RA é utilizada para adicionar módulos virtuais que podem interagir com hardwares dos laboratórios remotos de forma bidirecional. Em um estudo mais recente, o grupo de pesquisadores apresentaram uma solução para o desenvolvimento de LRA para o ensino de automação (Márquez et al., 2017). Por meio do LRA desenvolvido, o aluno pode programar um PLC (Controlador Lógico Programável) para automatizar uma planta de laboratório real, que é aumentada por objetos virtuais 3D que interagem com os reais (Figura 1)
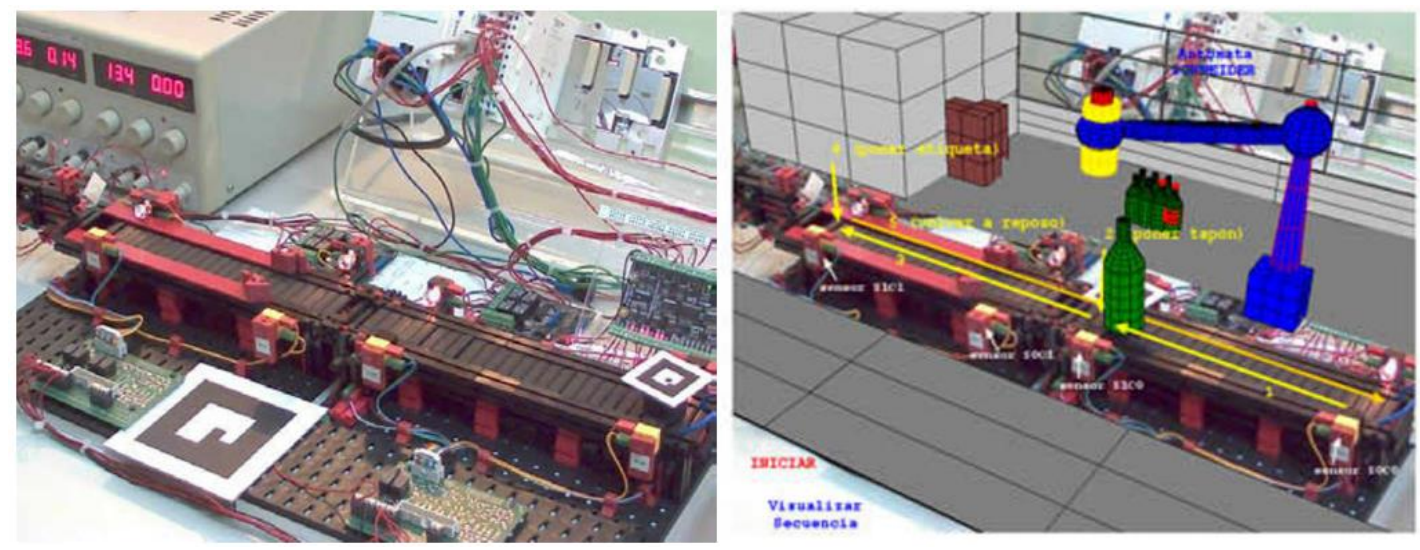

Figura 1 - Laboratório Remoto Aumento - PLC. Fonte: (Mejías et al., 2013)

Por meio da interface do computador, o aluno pode operar o PLC remoto, e observar a evolução do esquema real de acordo com a programação que ele enviou ao controlador (Márquez et al., 2017). Assim, os elementos reais e virtuais interagem uns com os outros, compartilhando o mesmo espaço tridimensional.

De forma semelhante, Rodriguez-Gil et al (2017) desenvolveram um laboratório híbrido que permite controlar um tanque de água virtual com uma placa FPGA. Aqui os pesquisadores privilegiaram uma arquitetura totalmente baseada na web, permitindo o acesso em qualquer plataforma que tenha um navegador compatível. Os autores aplicaram o laboratorio para uma turma de 58 alunos dos cursos de Gestão de Empresas e Engenharia Industrial, a fim de conhecer a opinião dos estudos em relação a ferramenta. Com isso, evidenciaram que a ferramenta tem potencial para envolver a atenção dos estudos, gerando resultados educacionais significativos.

Nesse sentido, Odeh, Shanab e Anabtawi (2015) realizaram um estudo comparativo entre os três tipos de laboratório práticos - laboratório híbrido, laboratório físico (hands-on) e laboratório virtual. Para isso, os autores desenvolveram um laboratório híbrido para práticas no ensino de circuitos elétricos. Os alunos do curso de engenharia elétrica da Universidade Técnica da Palestina realizaram o mesmo experimento usando as três formas de experimentação e fizeram uma avaliação comparativa por meio de um questionário. Os resultados mostraram que o laboratório remoto de RA foi, de modo geral, bem aceito pelos alunos, indicando que os mesmos perceberam esses laboratórios como de fácil utilização e entendimento, com maior flexibilidade em relação ao tempo e ao local de acesso e maior segurança na utilização.

\section{Procedimentos metodológicos}

O estudo exploratório faz parte de uma pesquisa ampla de caráter explicativo (causa e feito) e abordagem mista (quali-quantitativa). O design é quase-experimental, na medida em que se busca identificar se um tratamento específico influencia um resultado, mas 
valendo-se de uma amostra de conveniência. A variável independente é o uso de recursos tecnológicos utilizados para o ensino de circuitos elétricos e as variáveis dependentes são os possíveis benefícios educacionais gerados relacionados à motivação, engajamento, usabilidade e aprendizagem percebida, além da progressão dos alunos no que se refere a compreensão do conteúdo explorado por meio dos laboratórios online.

Para isso, foram desenvolvidos dois laboratórios online: um Laboratório Virtual de RA e um Laboratório Remoto Aumentado. Os recursos desenvolvidos foram utilizados como apoio para ensino de conceitos básicos de eletricidade no curso técnico em Eletromecânica na disciplina de Eletrônica, com 31 estudantes, como forma de revisão de conteúdo. O curso técnico em Eletromecânica é ofertado na modalidade concomitante ao ensino médio e a disciplina de Eletrônica é ministrada no $3^{\circ}$ ano do ensino médio de uma instituição pública federal localizada na cidade de Araranguá Santa Catarina.

A aplicação em sala de aula foi precedida por uma avaliação diagnóstica e finalizada com uma avaliação conceitual. Para essas avaliações foi utilizado o "Teste para verificar se o respondente possui concepções científicas sobre corrente elétrica em circuitos simples", desenvolvido por Silveira (2011). O teste é composto por 14 questões sobre os conceitos básicos de corrente elétrica e associação de resistores. Esse teste compõe os instrumentos de coleta de dados e teve como objetivo de identificar a progressão dos alunos no que se refere a compreensão do conteúdo explorado por meio dos laboratórios online. Nesse sentido, 26 alunos realizaram as duas avaliações diagnóstica e conceitual, portanto estes foram considerados para a análise.

Além disso, depois de todas as atividades finalizadas, foi aplicado o questionário "Modelo de Avaliação de Abordagens Educacionais em Realidade Aumentada Móvel" (Evaluation model of Mobile Augmented Reality Educational Approaches - MAREEA) para a avaliação dos recursos tecnológicos, desenvolvido por Herpich et al. (2019), com objetivo de conhecer as percepções dos estudantes em relação a motivação, engajamento, usabilidade e aprendizagem percebida ao utilizaram os laboratórios online. O questionário é composto por 37 questões dispostas em uma escala Likert e uma questão aberta para os alunos expressarem os pontos positivos e negativos percebidos. O questionário foi respondido por 19 alunos.

\subsection{O Laboratório Remoto Aumentada - Painel Elétrico}

O LRA foi desenvolvido a partir de um laboratório remoto já existente, disponibilizado pelo Laboratório de Experimentação Remota (Rexlab), vinculado à Universidade Federal de Santa Catarina (UFSC) - Campus Araranguá, o "Painel Elétrico AC" (Figura 2). Este experimento aborda as associações em série, paralela e mista em redes de corrente alternada. Nele é possível observar a intensidade luminosa de seis lâmpadas variar de acordo com a configuração do circuito, para isso quatro chaves são dispostas em diferentes pontos e controladas pelo usuário. 


\section{Painel Elétrico CA}
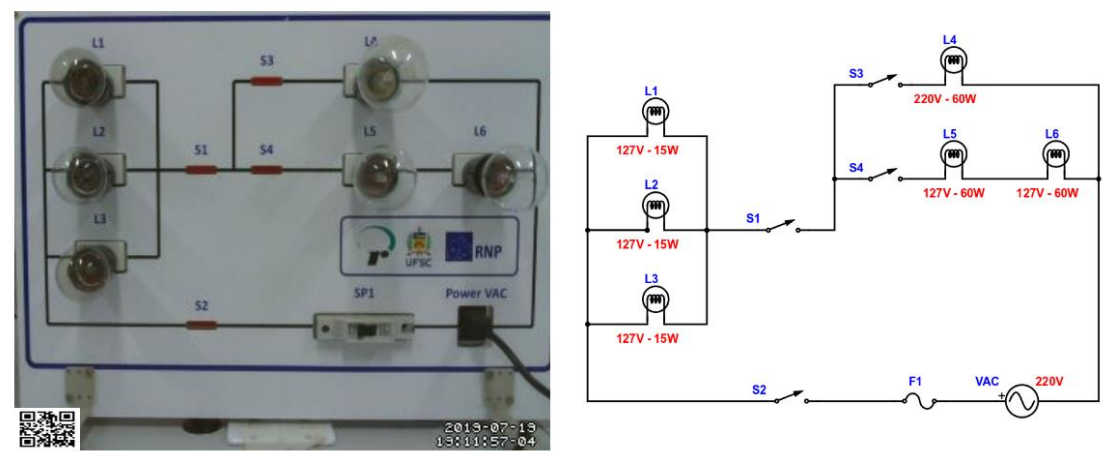

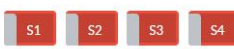

Figura 2 - Painel Elétrico CA disponibilizado pelo Rexlab. Fonte: relle.ufsc.br

A RA foi utilizada neste experimento para demonstrar de forma lúdica como a corrente elétrica percorre o circuito, mostrando a presença, ausência e sua intensidade por meio de animação e composição de cores. Além disso, também foram acrescentados os valores das correntes em cada ponto do circuito. Como marcador para a RA, é utilizado o próprio streaming de vídeo do experimento remoto. Assim, o estudante irá apontar seu smartphone para a tela do computador para visualizar e interagir com laboratório remoto aumentado. A figura 3 apresenta como ocorre acesso ao LRA "Painel Elétrico AC Aumentado".

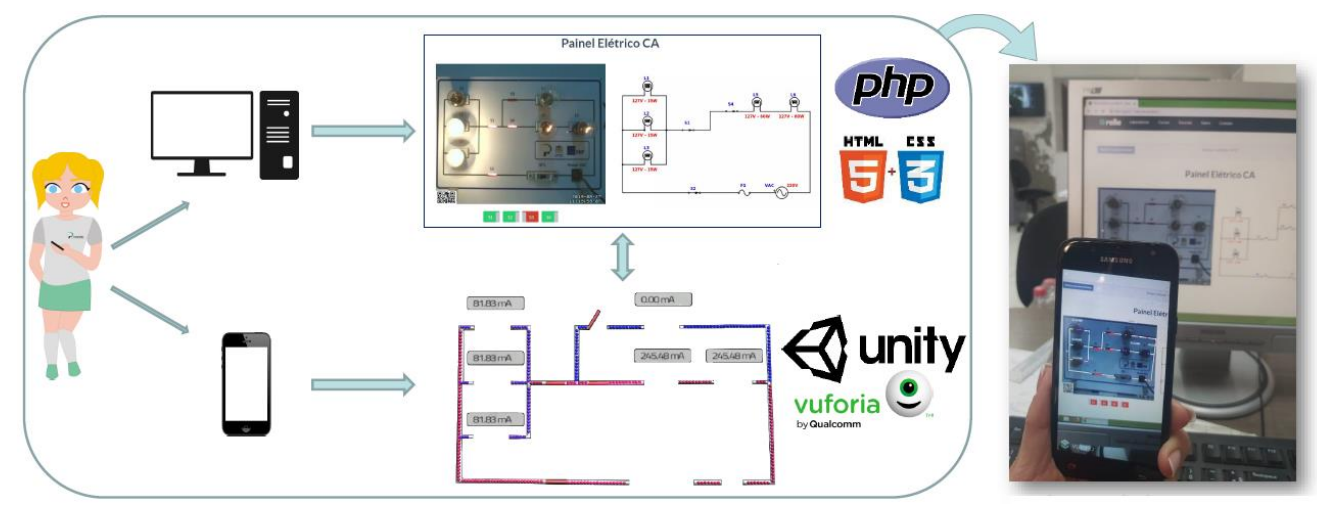

Figura 3 - Funcionamento do acesso ao LRA "Painel Elétrico AC Aumentado".

Os objetos 3D desenvolvidos para a composição do Laboratório Remoto Aumentado foram modelados por meio da ferramenta Unity 3D (2018), em linguagem de programa C\#. A RA é integrada por meio do o framework Vuforia, que por meio de uma série de abstrações, possibilita que se relacionem marcadores, desenvolvidos em Unity. Dessa forma, ao detectar por meio da câmera, a presença de um marcador conhecido, o algoritmo da API gera na tela o modelo tridimensional correspondente.

A comunicação bidirecional entre a RA e o hardware do laboratório remoto foi implementada através de um sistema de web sockets. Para tanto foi utilizada SocketIO, uma biblioteca Javascript/C\#. Mais detalhes sobre a arquitetura desenvolvida pode ser encontra em Nicolete et al., (2019).

O Laboratório Virtual de RA, de forma semelhante ao LRA, foi modelado e animado por meio da plataforma Unity 3D e o framework Vuforia para a integração das tecnologias de realidade aumentada. A RA desenvolvida consiste em três circuitos elétricos simples (Figura 3), dos quais os alunos podem interagir conectando e 
desconectando as lâmpadas e observando os diferentes comportamentos da corrente elétrica em circuitos em série (Figura 3b) e em paralelo (Figura 3c).

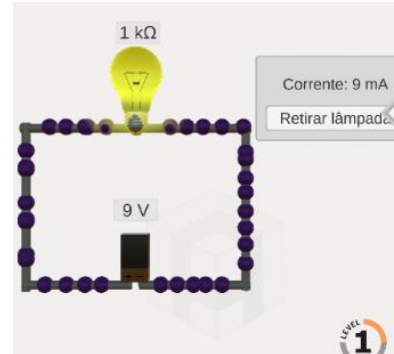

(a)

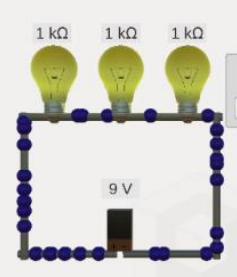

1

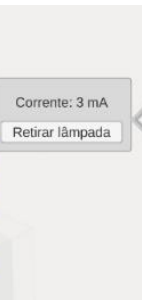

(2)

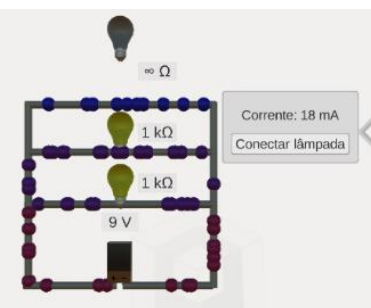

(3)

(b)

(c)

Figura 4 - Laboratório Virtual de RA - Circuitos Elétricos série e paralelo

O usuário tem acesso a RA do Laboratório Remoto Aumentado e o Laboratório Virtual de RA por meio do aplicativo avatAR-LRA, que pode ser encontrado na loja de aplicativo da Google - Play Store.

\subsection{Aplicação em sala de aula}

A aplicação ocorreu em 2 aulas presencias e uma atividade extraclasse. Nas aulas presenciais os alunos instalaram o aplicativo móvel (app), acompanharam as orientações gerais do professor e iniciaram suas atividades práticas.

Por meio do ambiente virtual de aprendizagem da instituição, foi disponibilizado aos alunos o material para realização das atividades, assim como em formato impresso, com as orientações para instalação e uso do aplicativo para acesso aos laboratórios, além do material pedagógico com três atividades.

A primeira atividade possuía uma breve revisão sobre o tema, o marcador para acesso ao Laboratório Virtual de RA e 3 questões que deveriam ser respondidas com ajuda das simulações. A segunda atividade era composta por duas questões, das quais os alunos precisavam responder com a ajuda do LRA. A primeira questionava sobre o tipo de associação que era apresentada no circuito elétrico do LRA e na segunda questão os alunos precisavam alterar as configurações do circuito, fechando as chaves 1 e 4 e, a partir da observação do comportamento luminoso das lâmpadas e do comportamento da corrente elétrica demostrada na RA, descrever o que estava acontecendo com o circuito elétrico. A terceira atividade era composta por 4 questões, que também deveriam ser respondidas a partir da realização do experimento no LRA e deveria ser realizada como uma atividade extraclasse, em um período de uma semana.

\section{Resultados e Discussão}

Com relação aos resultados obtidos pelo questionário MAREEA, a maioria das respostas ficaram entre 4 e 5, representando "4.Concordo Parcialmente" e "5.Concordo Totalmente", demostrando a percepção positiva dos alunos em relação ao uso de laboratórios online nos processos de ensino e aprendizagem. A Figura 5 apresenta a moda calculada para cada questão. 


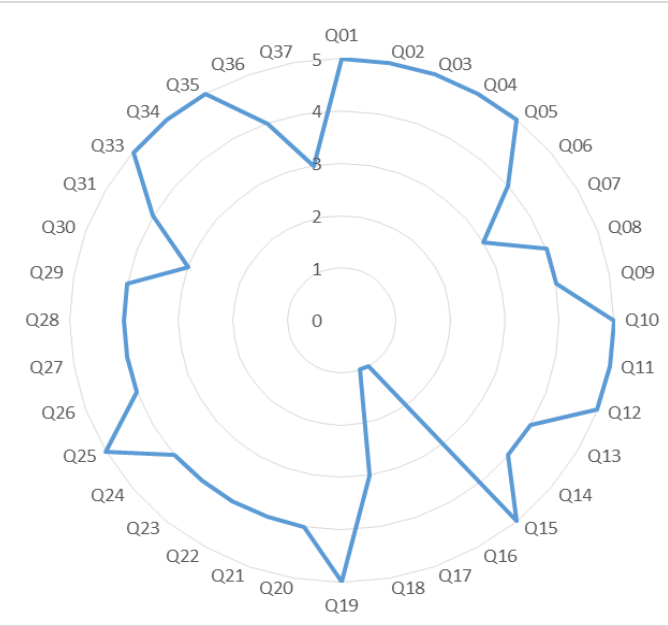

Figura 5 - Moda calculada para as frequências apresentadas para cada afirmativa do questionário MAREEA - Ensino Técnico

Abaixo são apresentados alguns relatos dos alunos sobre sua experiência:

- O aplicativo é algo útil para se entender as propostas técnicas das disciplinas do curso que envolvem eletricidade, e por ser um experimento virtual/digital não há riscos de acidentes, e você consegue entender como aquilo funciona mesmo sem tê-lo feito em forma de experimento físico (Aluno 7).

- Gostei muito do aplicativo educacional de realidade aumentada, pois me ajudou a entender um pouco mais sobre os circuitos. Foi uma experiência muito boa e algo novo na sala de aula, e com certeza ajuda muito a explicar o que acontece em um circuito (Aluno 8).

Entretanto, alguns alunos enfrentaram problemas ao utilizarem os laboratórios. Alguns smartphones apresentaram dificuldades para reconhecer os marcados de RA. Foi possível identificar que alguns smartphones, ao acessar a câmera fotográfica pelo aplicativo, apresentava uma perda na qualidade do foco da lente, o que impedia o reconhecimento do marcador pelo aplicativo. Para contornar essa situação, o professor da disciplina remanejou os grupos a fim de que cada grupo possuísse ao menos um smartphone que permitisse a utilização da ferramenta. Contudo, essa situação gerou frustação em alguns alunos que gostariam de realizar as atividades diretamente do seu celular. Abaixo alguns relatos dos estudantes:

- Melhorar o aplicativo, extremamente difícil de focar utilizando a câmera do app, o que prejudica na hora de realizar os exercícios (Aluno 12).

- A ideia é muito boa, o aplicativo tem ótimo uso educacional e tem grande potencial didático, porém, a interface poderia ser mais atraente. Um problema encontrado é a dificuldade do celular reconhecer a imagem, isso faz o usuário passar um bom trabalho para realizar a simulação (Aluno 16).

Ainda assim, de modo geral, os alunos tiveram uma postura positiva em todos os fatores de qualidade pesquisados, como pode ser observado na Tabela 1. A média de todas as dimensões foi de 3,84 pontos, com desvio padrão (DP) de 1,15. E os fatores que obtiveram maiores índices de concordância foram usabilidade (Média: 4,00 - DP: 1,10) e aprendizagem (Média: 3,90 - DP: 1,13). 
Tabela 1 - Médias e desvio padrão das percepções dos estudantes em relação a Usabilidade, Engajamento, Motivação e Aprendizagem

\begin{tabular}{|c|c|c|c|c|c|c|c|c|}
\hline \multirow{3}{*}{ Pesquisa } & \multirow{3}{*}{ Fatores } & \multirow{3}{*}{ Média } & \multirow{3}{*}{$\begin{array}{l}\text { Desvio } \\
\text { padrão }\end{array}$} & \multicolumn{5}{|c|}{ Percepção } \\
\hline & & & & \multicolumn{4}{|c|}{ Baixo } & Alto \\
\hline & & & & 1 & 2 & 3 & 4 & 5 \\
\hline \multirow[t]{4}{*}{ MAREEA } & Usabilidade & 4,00 & 1,10 & & & & & \\
\hline & Engajamento & 3,69 & 1,34 & & & & & \\
\hline & Motivação & 3,76 & 1,03 & & & & & \\
\hline & Aprendizagem & 3,90 & 1,13 & & & & & \\
\hline
\end{tabular}

A dimensão de usabilidade pretendia verificar o quão intuitivo e fácil é para os usuários aprenderem a usar e interagir com os laboratórios online (Herpich et al., 2019). Desse modo, os alunos concordaram que a forma de usar o app é de fácil entendimento e que foi simples aprender utilizá-lo. Entretanto, ao serem questionados se o aplicativo é fácil de usar, $5 \%$ dos estudantes discordaram com essa afirmação e $21 \%$ preferiram não opinar. Assim, evidencia-se que apesar de o aplicativo ser de fácil utilização, as dificuldades no reconhecimento dos marcadores tornaram dispendiosa a sua utilização.

Contudo, os estudantes reconheceram que os laboratórios online representam ganho em seu processo de aprendizagem (Figura 6). Para eles, as experiências nos laboratórios online foram apropriadas $(\mathrm{Q} 34)$ e úteis $(\mathrm{Q} 28)$ para o seu aprendizado, além de permitir interagir com simulações que dificilmente realizariam no mundo real (Q33).

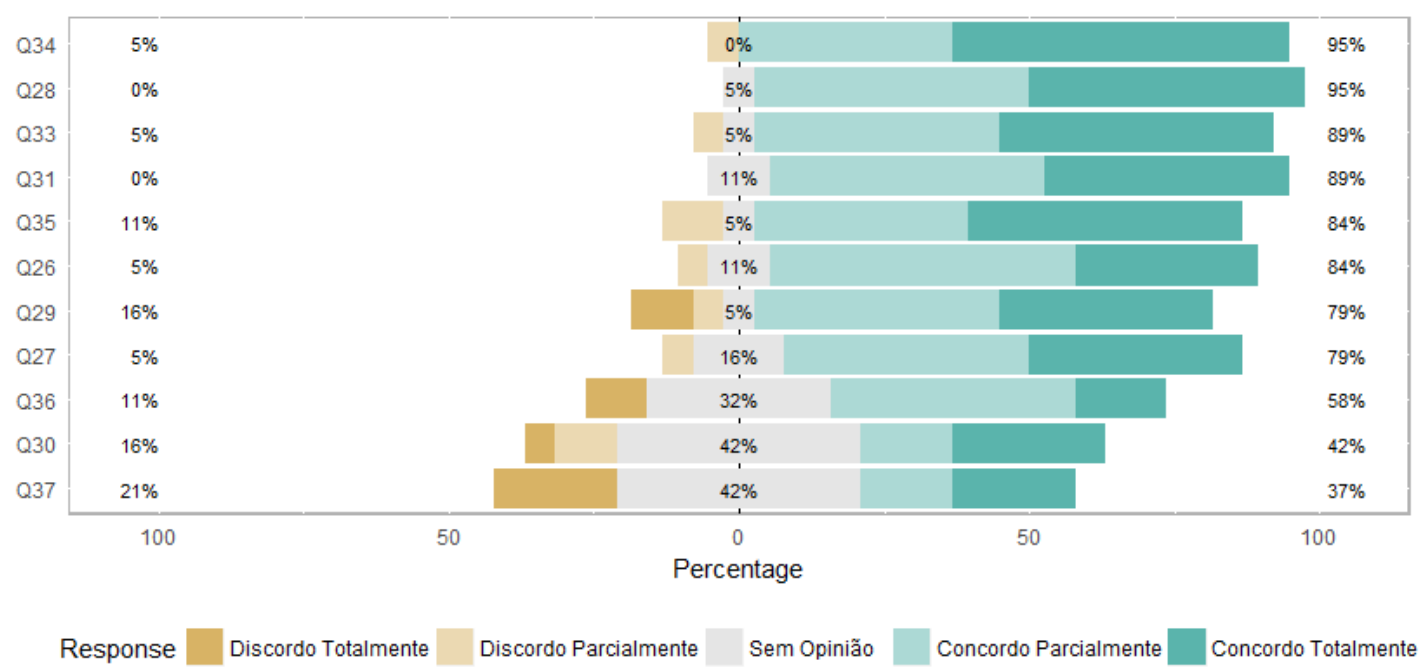

Figura 6 - Percepção dos estudantes do ensino técnico em relação a sua aprendizagem com o uso da Realidade Aumentada e do Laboratório Remoto Aumentado

A dimensão de motivação foi a dimensão em que os problemas de reconhecimento dos marcadores mais afetou. A questão "Q25. Eu realmente gostei de estudar com este aplicativo de realidade aumentada" apresentou apenas $58 \%$ de aceitação. Ainda assim, os índices de concordância foram maiores em relação aos níveis de discordância em todas as questões deste construto. Destaca-se ainda a questão "Q19. Está claro para mim como o conteúdo educacional destes aplicativos de realidade aumentada estão relacionados as coisas que conheço.", que apresentou o maior índice de concordância (89\%) deste construto, o que demostra que o conteúdo é apresentado de forma significativa, estabelecendo conexão do tema abordado com experiências anteriores dos alunos. 
O menor índice apresentado pelos alunos foi em relação ao Engajamento. Apesar de os alunos concordarem que o conteúdo educacional apresentado no aplicativo é relevante, eles não se sentiram totalmente envolvidos com a atividade de aprendizagem. A questão "Q16. Eu estava tão envolvido na tarefa com este aplicativo de realidade aumentada que perdi a noção do tempo." e a "Q17. Eu ignorei as coisas ao meu redor quando eu estava usando este aplicativo de realidade aumentada", obtiveram os maiores índices de discordância, totalizando $47 \%$ e 58\%, respectivamente.

Com os resultados das avaliações diagnóstica e conceitual foi possível, ainda que de forma preliminar, realizar algumas análises em relação a progressão dos alunos no que se refere a compreensão do conteúdo explorado com apoio dos laboratórios online. Vale ressaltar que o tamanho da amostra representa uma limitação deste estudo. Contudo, essa análise atende aos objetivos propostos, que é de realizar um estudo exploratório sobre os possíveis potenciais dessas tecnologias para a aprendizagem.

Diante disso, foi traçado uma média geral sobre o aproveitamento dos alunos. A média inicial referente à Avaliação Diagnóstica $(\mathrm{AD})$ que foi de 3,11 , já na Avaliação Conceitual (AC) os alunos obtiveram uma média de 4,30 (Tabela 2). Aplicando o Teste t de Student, pode-se afirmar que o resultado obtido foi significativo estatisticamente, uma vez que $\boldsymbol{p}$ apresentou valor de $0,001(\alpha=0,05)$.

Tabela 2 - Médias e valores do teste t de Student para as avaliações diagnóstica e conceitual

\begin{tabular}{|l|l|l|l|l|l|}
\hline $\boldsymbol{\mu}$ AD & Desvio padrão AD & $\boldsymbol{\mu}$ AC & Desvio padrão AC & Valor de $\boldsymbol{t}$ & Valor de $\boldsymbol{p}$ \\
\hline 3,11 & 1,64 & 4,30 & 1,58 & 3,751 & 0,001 \\
\hline
\end{tabular}

*Nível de significância de 5\% $(\alpha=0,05)$

Por meio do gráfico boxplot, representado pela Figura 7, é possível analisar detalhadamente os dados. Assim, percebe-se que na avaliação diagnóstica o aproveitamento da turma ficou entre 0,7 e 5,0, com uma distribuição mais concentrada e simétrica que aquelas da avaliação conceitual, além de um menor valor mediano $(2,9)$ (Tabela 3). Por outro lado, as notas da AC ficaram entre 2,9 e 6,4, e apontam um maior valor mediano $(3,6)$, com uma distribuição menos concentrada que a $\mathrm{AD}$.

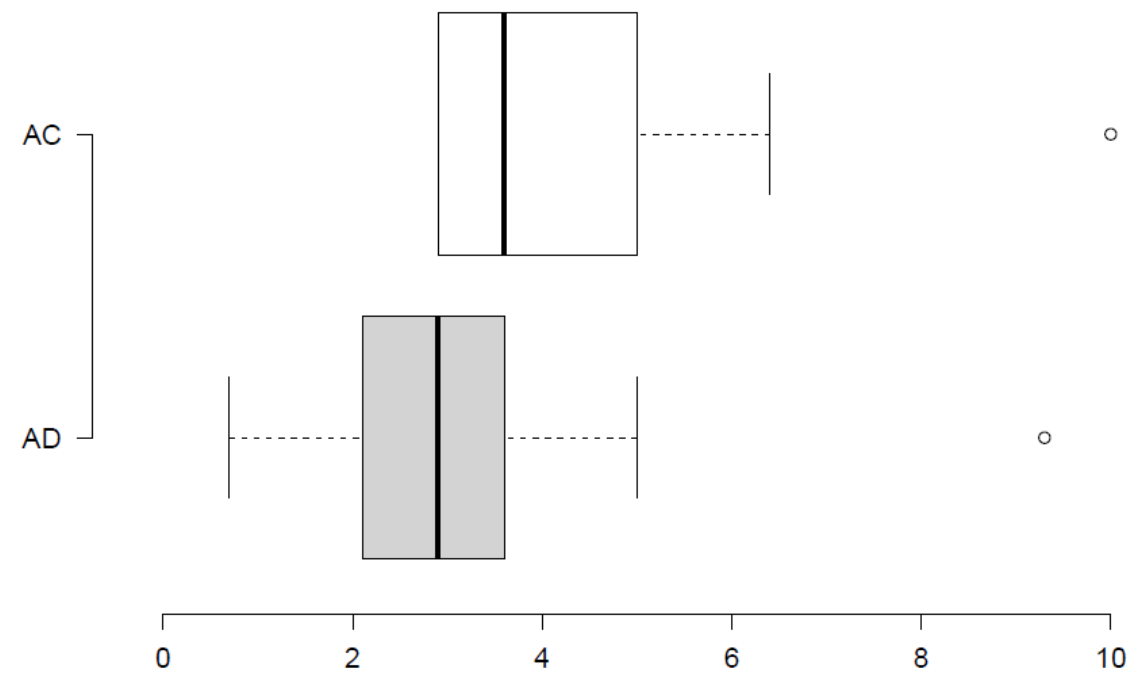

Figura 7 - Boxplot das notas obtidas pelos alunos nas avaliações diagnóstica e conceitual *As linhas centrais mostram as medianas; os limites da caixa indicam os percentis 25 e 75 , conforme determinado pelo software $\mathrm{R}$; os traços estendem 1,5 vezes a faixa interquartil dos percentis 25 e 75 , os valores extremos são representados por pontos. $\mathrm{n}=26$ pontos de amostra. 
Tabela 3 - Estatísticas do gráfico Boxplot (Figura 5)

\begin{tabular}{|l|c|c|}
\hline & Avaliação Diagnóstica - AD & Avaliação Conceitual - AC \\
\hline Máximo & 5.00 & 6.40 \\
\hline $\mathbf{3}^{\mathbf{0}}$ quartil & 3.60 & 5.00 \\
\hline Mediana & 2.90 & 3.60 \\
\hline $\mathbf{1}^{\mathbf{0}}$ quartil & 2.10 & 2.90 \\
\hline Mínimo & 0.70 & 2.90 \\
\hline $\mathbf{N}^{\mathbf{0}}$ pontos da amostra & 26 & 26 \\
\hline
\end{tabular}

Ainda, evidencia-se que a avaliação conceitual tem a mediana mais próxima do primeiro quartil, o que identifica que os dados são positivamente assimétricos. Desse modo, verifica-se que a turma obteve um progresso na compreensão do conteúdo explorado, a maioria das notas ficaram acima das notas mais altas da avaliação diagnóstica. Entretanto, vale ressaltar que em ambas avaliações os alunos não obtiveram notas muito altas, e alguns alunos não apresentaram progressão entre $\mathrm{AD}$ e $\mathrm{AC}$. É importante destacar que esse conteúdo já havia sido explorado naquele semestre e os laboratórios foram utilizados para uma revisão de conteúdo.

\section{Considerações finais}

Realizadas as atividades junto aos alunos do ensino médio técnico em Eletromecânica, percebeu-se uma atitude positiva dos estudantes com a utilização dos laboratórios online em sala de aula. Esses resultados corrobora com os resultados obtidos por outros pesquisadores, tais como Odeh, Shanab e Anabtawi (2015), Rodriguez-Gil et al (2017) e Andújar Mejias e Marquez (2011), os quais também apresentaram a percepção dos alunos quanto ao uso dessas tecnologias, e obtiveram como resultado a fácil utilização e entendimento, motivando-os a realizarem as atividades propostas.

Os problemas técnicos encontrados devido à tecnologia utilizada por alguns estudantes provocam frequentemente uma compreensão adversa sobre o que é falha do processo no ensino e da aprendizagem e do que é limitação do recurso tecnológico utilizado. Esta situação pode alterar a percepção, motivação e o engajamento do aluno nas atividades propostas. Por outro lado, a maioria dos alunos apresentaram resultados satisfatórios no que tange a aprendizagem com uso dos LRA e LVRA, o que significa que, seja de forma ubíqua ou completamente consciente, estes recursos de fato colaboram para o processo de ensino e aprendizagem no que tange a prática de conteúdos que requerem tal estímulo sinestésico.

Para trabalhos futuros, pretende-se realizar melhorias no aplicativo referentes ao desempenho no reconhecimento de marcadores e aprimorar a interface do usuário, inserindo informações de uso, como tutoriais e conteúdos educacionais que podem servir de auxílio durante a experimentação. Após as melhorias, pretende-se expandir o uso desses recursos para outras turmas.

\section{REFERÊNCIAS}

ANDÚJAR, J. M.; MEJIAS, A.; MARQUEZ, M. A. Augmented reality for the improvement of remote laboratories: An augmented remote laboratory. IEEE Transactions on Education, v. 54, n. 3, p. 492-500, 2011.

AZUMA, R. et al. Recent advances in augmented reality. NAVAL RESEARCH LAB WASHINGTON DC. 2001

FRANK, J. A.; KAPILA, V. Mixed-reality learning environments: Integrating mobile interfaces with laboratory test-beds. Computers \& Education, v. 110, p. 88-104, 2017.

V. $17 \mathrm{~N}^{\mathrm{o}}$ 3, dezembro, 2019

RENOTE DOI: 
HERPICH, F. et al. Modelo de avaliação de abordagens educacionais em realidade Aumentada Móvel. RENOTE, v. 17, n. 1, p. 355-364, 2019.

INEP/MEC. Censo Escolar Brasileiro 2018. Brasil, 2018. Disponível em: < http://portal.inep.gov.br/basica-censo $>$.

MÁRQUEZ, M. et al. Programming and testing a PLC to control a scalable industrial plant in remote way. Proceedings of 2017 4th Experiment at International Conference: Online Experimentation, exp.at 2017, 2017. Conference Paper. p.105-106.

MEJÍAS, A.; ANDÚJAR, J. M.; SÁNCHEZ, M. A. M. Digital electronics augmented remote laboratory: DEARLab. International Journal of Engineering Education, v. 30, n. 4, p. 950963, 2014.

MEJÍAS, A. et al. A complete solution for developing remote labs. IFAC Proceedings Volumes (IFAC-PapersOnline), 2013. Conference Paper PART 1. p.96-101.

NICOLETE, P. et al. Laboratórios Remotos Aumentados para o ensino de Circuitos Elétricos. Anais do Workshop de Informática na Escola, 2019. p.1104.

ODEH, S.; SHANAB, S. A.; ANABTAWI, M. Augmented Reality Internet Labs versus its Traditional and Virtual Equivalence. International Journal of Emerging Technologies in Learning, v. 10, n. 3, 2015.

RODRIGUES, J. et al. Adding augmented reality to laboratory experimentation. Proceedings of 2017 4th Experiment at International Conference: Online Experimentation, exp.at 2017, 2017. Conference Paper. p.135-136.

RODRIGUEZ-GIL, L. et al. Towards new multiplatform hybrid online laboratory models. IEEE Transactions on Learning Technologies, v. 10, n. 3, p. 318-330, 2017.

SILVEIRA, F. L. Um teste para verificar se o respondente possui concepções científicas sobre corrente elétrica em circuitos simples. Publicado em Física no ensino médio: falhas e soluções. Organizador: Rocha Filho, JB, Porto Alegre: Edipucrs, p. 61-67, 2011.

ZANDAVI, S. M.; CHUNG, V. Augmented Reality for Remote Laboratory Improving Educational Learning: Using Elevated Particle Swarm Optimization in Object Tracking Scheme. 2018 International Joint Conference on Neural Networks (IJCNN), 2018. IEEE. p.1-6. 